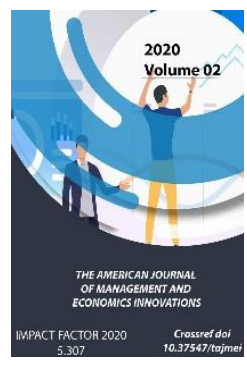

\title{
Purchaser Attributes And Their Impact On Tolerating Internet Shopping, With Regards To Various Item Types
}

\author{
Dimitrios Sarigiannidis \\ Department Of Business Administration, School Of Business And Economics, Kavala Institute \\ Of Technology Agios Loukas, Greece
}

\section{ABSTRACT}

Web based shopping is among the most famous exercises of the web, yet the reasons why buyers purchase online are as yet hazy. In spite of the fact that it is inferred that purchaser acknowledgment of web based shopping is influenced by various items very few examinations have embraced this view. This examination endeavors to analyze buyers' disposition when making on the web buys with regards to various item types. A hypothetical system is proposed dependent on the determinants of buyer conduct and client acknowledgment of internet shopping, just as online item grouping. The elements that were chosen to be tried are Close to home Ingenuity of Data Innovation, Self-adequacy, Seen security, Protection, Item association and how they influence shopper demeanor towards internet shopping. Connection examination, from the outset, to decide the connections among the factors and relapse investigation a short time later to confirm the degree of the variable cooperation were utilized to test the theories. In view of the previously mentioned investigations, results were attracted and contrasted with the outcomes found by Lian and Lin in a comparative report. It has been discovered that PIIT, saw security and item association affect the demeanor towards web based shopping, yet the outcomes fluctuate among the diverse item types.

\section{KEYWORDS}

Individual ingenuity of data innovation, self - adequacy, seen security, protection concerns, item contribution 


\section{INTRODUCTION}

The USA, online retail deals of 2019 expanded by $2.1 \%$ more than 2018, arriving at an aggregate of $\$ 145$ billion dollars, and from 2012 to 2019 retail e-deals expanded on normal yearly development pace of $18.1 \%$. In the European Association of the 27 individuals, 37\% of the web clients have made an online buy in 2019, a 5\% expansion over the earlier year. In the Assembled Realm, Denmark, the Netherlands, Norway and Sweden over $60 \%$ of the web clients have made an online buy, though the identical number in Greece, Lithuania, Bulgaria and Romania is under $10 \%$. As it very well may be induced from the abovementioned, the size of internet shopping selection fluctuates between the created and non-industrial nations .Understanding the open doors this new market has to bring to the table is essential for any business that needs to partake in it and be serious. In addition, online purchaser demeanor is an issue that worries numerous analysts. A basic inquiry around there is, which are the components that decide shoppers' choice to make a buy from a specific electronic shop. Finding the attributes of potential purchasers can assist endeavors with precisely discovering potential objective business sectors.

\section{Hypothetical Foundation}

The web is growing quickly and keeping in mind that its ubiquity is developing, an ever increasing number of clients become acquainted with it and receive it as a medium to look for data and shop on the web. This part sums up the determinants that build the shopper conduct, the elements that decide the client acknowledgment of web based shopping and a short audit of recently directed explores concerning the previously mentioned.

\section{Determinants of buyer conduct}

Purchaser conduct is influenced by four classifications of variables: social components, social elements, individual elements and mental elements. The principal classification of social components, incorporates terms, for example, culture, subculture and social class. The term culture is intricate and includes the information, convictions, expressions, laws, morals, customs and numerous different capacities and propensities that are gotten by an individual just by being important for the general public.

The manner in which an inspired individual acts is impacted by his/her impression of the given circumstance. The biggest piece of human conduct is found out. It is said that an individual's learning is created through the connection of thought processes, improvements and responses. Through acting and learning individuals structure convictions and mentalities that influence their buying conduct. Convictions are the enlightening way an individual considers something and depend on information, assessment or confidence and may include wistful charges, while disposition respects the ceaseless assessment, the feelings and the propensities of an individual towards an article or thought

Self-adequacy Web self-viability gets from the social intellectual hypothesis proposed by Bandura. Inside this point of view, one's conduct is continually under proportional impact from psychological (and other individual factors, for example, inspiration) and natural impacts. Bandura calls this three-path association of conduct, psychological components, and ecological circumstances the "triadic correspondence". All in all, self-viability in web based shopping depicts the person's capacity to apply their abilities to finish a buy on the web. Moreover,showed that individual web self-adequacy positively affects client 
acknowledgment of web based shopping. buyers who have low self-viability levels are unreliable and feel awkward making buys over the web

\section{CONCLUSION}

From all the abovementioned, it is clarified that distinctive item types are liable for the separations of the outcomes. As a last end it tends to be said that purchaser disposition towards internet shopping is influenced essentially by the item being referred to. Also, it tends to be said that in Greece individuals are as yet trying different things with online buys despite the fact that the yearly development rate is higher than half. In the Nielsen Worldwide Buyer Report it is expressed that $23 \%$ of the Greek online customers didn't plan to make any buys in the accompanying a half year, when the Europe's normal was close to $21 \%$. In a similar report it has been discovered that Greek online customers favor electronic gear and PC equipment which fall under the significant expense, infrequently bought, substantial products which can legitimize the reluctance of online customers to buy online present moment. Generally, clearly the item order and sort of items that were chosen are answerable for the varieties in the outcomes in the current investigation. Because of the various qualities each item has, purchasers' demeanor shows varieties. Purchasers act contrastingly when purchasing reasonable items and diversely when they are purchasing costly items. Likewise, their mentality changes when it includes ordinary items and when they purchase items and administrations they plan to use in the long haul. The current investigation gives a comprehension of what drives shoppers to purchase their items on the web and can be utilized by organizations that advance their items through the web. Nonetheless, no close to home apparent qualities, for example, seen comfort, seen threat, seen site quality and saw benefits, that could change the discoveries of the exploration, were raised.

\section{REFERENCES}

1. Zinkhan, G. M., (2016). Promoting Standards and Practice. London: Pitman Distributing.

2. Chaipoopirutana, S. and Combs, H., (2010). A calculated and operational meaning of individual creativity in the space of data innovation. Data Frameworks Exploration, 41 (2), 144155.

3. C. H. and Kim, Y. G., (2013). Mentalities, fulfillment and utilization: Elements adding to each in the acknowledgment of data innovation. Conduct and Data Innovation, 25 (8), 337-341.

4. Fenech, T., (2013).Dependability in electronic business: The part of protection, security, and site credits. Diary of Vital Data Frameworks, 44, 185-190.

5. Mathews, S. and Healy, M., (2017). Breaking the Fantasies of Remunerations: An Exploratory Investigation of Perspectives about Information Sharing. Data Assets The executives Diary, 35 (4), 314 - 321.

6. Tractinskky, N.(2011). Impact of normal practices, seen energy and web based shopping nervousness on clients' reception of online retail shopping: An experimental examination in the 
The American Journal of Management and Economics Innovations (ISSN - 2693-0811)

Published: January 08, 2021 | Pages: 1-4

Doi: https://doi.org/10.37547/tajmei/Volumeo3lssue01-01

Turkish setting. Global Diary of Retail and Dispersion The board, 11 (4), 110113.

7. Cheung, M. T., (2019). Purchaser impression of interface quality, security, and devotion in electronic business. Data and The executives, 35, 741-747. 